\title{
Nuclear bodies and compartmentalization of pre-mRNA splicing factors in higher plants
}

Received: 30 October 2003 / Revised: 10 December 2003 / Accepted: 10 December 2003 / Published online: 23 January 2004 (C) Springer-Verlag 2004

\begin{abstract}
We studied the fine structural organization of nuclear bodies in the root meristem during germination of maize and Arabidopsis thaliana using electron microscopy (EM). Cajal bodies (CBs) were observed in quiescent embryos and germinating cells in both species. The number and distribution of $\mathrm{CBs}$ were investigated. To characterize the nuclear splicing domains, immunofluorescence labelling with antibodies against splicing factors (U2B" and m3G-snRNAs) and in situ hybridisation (with U1/U6 antisense probes) were performed combined with confocal microscopy. Antibodies specific to the Arabidopsis SR splicing factor atRSp31 were produced. AtRSp31 was detected in quiescent nuclei and in germinating cells. This study revealed an unexpected speckled nuclear organization of atRSp31 in root epidermal cells where micro-clusters of interchromatin granules were also observed by EM. Therefore, we examined the distribution of green fluorescent protein (GFP)-tagged atRSp31 in living cells after Agrobacterium -mediated transient expression. When expressed transiently, atRSp31-GFP exhibited a speckled distribution in leaf cells. Treatments with $\alpha$-amanitin, okadaic acid, staurosporine or heat shock induced the speckles to reorganize. Furthermore, we generated stable Arabidopsis transgenics expressing atRSp31-GFP. The distribution of the fusion protein was identical to that of endogenous atRSp31. Three-dimensional time-lapse confocal microscopy showed that speckles were highly dynamic domains over time.
\end{abstract}

Communicated by P. Shaw

\begin{tabular}{l}
\hline S. Docquier and P. Motte contributed equally to this work \\
\hline S. Docquier · V. Tillemans $\cdot$ R. Deltour $\cdot$ P. Motte ( \\
Laboratory of Plant Cell Biology, Department of Life Sciences, \\
University of Liège, \\
Bld du Rectorat, 27 Sart Tilman, 4000 Liège, Belgium \\
e-mail: patrick.motte@ulg.ac.be
\end{tabular}

\section{Introduction}

Splicing of nuclear pre-mRNA takes place within a multicomponent ribonucleoprotein (RNP) complex called the spliceosome, which contains the small nuclear ribonucleoprotein particles (snRNPs) U1, U2 and U4/ U5/U6 as well as non-snRNP proteins (Kramer1996). The non-snRNP serine/arginine-rich splicing factors (SR proteins) are involved in multiple steps of constitutive and alternative splicing (Lorkovic and Barta2002). The SR proteins constitute a family of splicing factors that are highly conserved throughout the metazoa (Graveley2000). In Arabidopsis thaliana a growing number of SR proteins have recently been uncovered (Lopato et al.1996, 1999a, 1999b,2002; Lorkovic and Barta2002; Savaldi-Goldstein et al.2003).

Splicing factors frequently exhibit local accumulation in subnuclear domains (Boudonck et al.1999; Eils et al.2000; Dundr and Misteli2001; Platani et al.2002). In mammalian cells, snRNPs have been detected as a diffuse nucleoplasmic pool and within the so-called speckles (or splicing-factor compartments) (Misteli2000; Sleeman et al.2001). In many cell types, snRNPs also accumulate in small spherical nuclear structures termed coiled bodies, recently renamed Cajal bodies (CBs) (Boudonck et al.1999; Gall2000,2001; Acevedo et al.2002; Hebert et al.2002; Ogg and Lamond2002; Platani et al.2002). Cajal bodies are involved in coordinating the assembly of snRNPs (Lamond and Earnshaw1998; Lewis and Tollervey2000; Darzacq et al.2002; Jady et al.2003). By immunofluorescence microscopy, SR proteins can be observed to colocalize with snRNPs within the speckles corresponding in electron microscopy (EM) to interchromatin granule clusters (IGCs) and perichromatin fibrils. Speckles appear as storage and assembly sites of spliceosomal components and the relocation of SR factors from speckles to actively transcribed genes is dependent on their phosphorylation status (reviewed in Misteli2000).

Despite considerable progress in the understanding of the compartmentalization of mammalian nuclei, the functional nuclear organization of plant cells remains to 
be defined. Microscopic investigations have shown the presence of different nuclear bodies and a compartmentalization of spliceosomal components as well (Beven et al.1995; Shaw1996; Boudonck et al.1998,1999; Straatman and Schel2001; Acevedo et al.2002; Cui and Moreno Diaz De La Espina2003). In the present study, we looked at the nuclear organization of splicing factors during germination of Zea mays and A. thaliana, two evolutionarily distant species. The embryo emerging from quiescence undergoes postembryonic vegetative development and the root meristem resumes growth first (Bewley1997). The quiescent nucleus resumes sequentially all its metabolic activities with the onset of seed imbibition (Deltour1985; Bewley1997). Germination is thus particularly convenient for observing putative modifications in spliceosomal factor relocation. One of the most interesting observations is a surprising speckled organization of the Arabidopsis SR splicing factor atRSp31 in differentiated root cells like trichoblasts and atrichoblasts. This was an unexpected result since IGC reservoirs of SR factors in mammalian cells have never been described in plants. To study further the distribution of plant SR proteins, we analysed the dynamics of atRSp31 fused to the green fluorescent protein (atRSp31GFP) in living cells after Agrobacterium -mediated transient expression and in stably expressing Arabidopsis transgenics. This is the first detailed analysis of the distribution and dynamics of a plant SR splicing factor; the nature of the speckles in plant cells is discussed.

\section{Materials and methods}

Plant germination and growth conditions

Maize ( Z. mays L. var. Orellana and var. Dione) and A. thaliana (ecotype Columbia) seeds were germinated in Petri dishes and incubated in a controlled temperature chamber in darkness at $16^{\circ} \mathrm{C}$ (maize) and in continuous illumination at $20^{\circ} \mathrm{C}$ ( Arabidopsis). For immunocytochemistry, Arabidopsis quiescent embryos were obtained by cutting the seed coats with a scalpel and peeling them off. For transient transformation, A. thaliana and Nicotiana tabacum (cv. Petit Havana) seeds were sown on soil in culture chamber and allowed to germinate and grow under long-day conditions (16 h light $/ 8 \mathrm{~h}$ darkphotoperiod) at $\sim 20^{\circ} \mathrm{C}$. Healthy Arabidopsis plants with well-developed leaf rosette and $20 \mathrm{~cm}$ height tobacco plants were used for Agrobacterium -mediated transient expression.

RNA extraction and reverse transcriptase-polymerase chain reaction (RT-PCR)

For transcript analysis, tissues were harvested and immediately frozen in liquid nitrogen. Total RNA was extracted using the guanidium method. cDNA for RT-mediated PCR was generated using SuperScript II Reverse Transcriptase (Invitrogen) according to the manufacturer's instructions. The primers used for RT-PCR analysis were as follow: for maize actin 1 (Accession no. J01238) $\left(5^{\prime}\right.$-ATGGCTGACGAGGATATCCAGCC-3') and (5'-GAAGCACTTCATGTGGACAATGCC-3'); for HAT-B-p50 (Accession no. AF171927) (5'-ATGGCGCTCAAGCAGAAGGAC- $\left.3^{\prime}\right)$ and (5'-TTGAACCACCAGCTCTGTCATC-3'); for B1-type cyclin (CycZme1, Accession no. U66607) (5'-ATGGAGTGCGCAAGGGAGTTG- $\left.3^{\prime}\right)$ and (5'-ACCTGACAACAGCTTCTTGTCAG- $3^{\prime}$ ). The PCR products were visualized on $1.0 \%$ agarose gels using a
UV light transilluminator. To confirm the RT-PCR specificity, the generated PCR products were subcloned into pGEM-T (Promega) and subsequently sequenced.

Expression vectors and polyclonal antibody production

The coding sequence of atRSp31 (Accession no. X99435) (Lopato et al.1996) was amplified by RT-PCR using a $5^{\prime}$ primer $\left(5^{\prime}-\right.$ ACATGCATGCGGCCAGTGTTCGTC- $\left.3^{\prime}\right)$ and $3^{\prime}$ primer $\left(5^{\prime}\right.$ GAAGATCTAGGTCTTCCTCTTGGGAC-3') containing Sph I and $B g l$ II restriction sites (italicised), respectively. To produce polyclonal antibodies against atRSp31, the PCR product was subcloned into the bacterial expression vector pQE70 (Qiagen) and named pQE-atRSp31 for recombinant protein production. The construct was verified by DNA sequencing. The fusion protein containing a C-terminal (His) 6 -tag was overexpressed in the Escherichia coli XL1Blue strain and purified according to the manufacturer's manual using Ni-NTA metal-affinity resin (Qiagen). Three rabbits were immunized with the purified (His) 6 -tag atRSp31. In order to test the specificity of the sera, immunoblotting was performed. Plant proteins were extracted in $0.1 \mathrm{M}$ TRIS-HCl, $\mathrm{pH} 8.3,0.5 \mathrm{M} \mathrm{NaCl}, 5 \mathrm{mM}$ dithiothreitol and $5 \mathrm{mM}$ EDTA, containing protease inhibitor $(2 \mathrm{mM}$ phenylmethylsulphonyl fluoride). Protein extracts were separated by SDS-polyacrylamide gel electrophoresis and blotted on PVDF membrane (Roche). Immunoblots were successively incubated with protein A purified IgG or affinity purified antibodies (Burke et al.1982): anti-atRSp31 at a 1:500 dilution and anti-rabbit $\operatorname{IgG}$ conjugated with alkaline phosphatase (Sigma) at a 1:3000 dilution followed by the detection of proteins using 5-bromo-4-chloro-3-indolyl phosphate-nitroblue tetrazolium reagents (Roche).

Binary vector construction

For construction of the pBAR35S::atRSp31-GFP plasmid, the atRSp31 and EGFP full-length coding sequences were first PCR amplified using Pfu polymerase (Promega) from pQE-atRSp31 (see above) with the $5^{\prime}$ primer (5'-ATGAGGCCAGTGTTCGTCGGC$\left.3^{\prime}\right)$ and the $3^{\prime}$ primer (5'-GAAGATCTTCCGGAAGGTCTTCCTCTTGG- ${ }^{\prime}$ ) containing a $B g l$ II site (italicised) and pEGFP (Clontech; Accession no. U76561) with the $5^{\prime}$ primer $\left(5^{\prime}\right.$-GAAGATCTATGGTGAGCAAGGGCGAGGAG- $3^{\prime}$ ) containing a $B g l$ II site, and the $3^{\prime}$ primer $\left(5^{\prime}\right.$-TTACTTGTACAGCTCGTCCATGC$\left.3^{\prime}\right)$, respectively. The resulting PCR fragments were digested with $B g l$ II and ligated. In this construction process, a linker of four amino acids (Ser-Gly-Arg-Ser) was inserted between the atRSp31 and GFP coding sequences. The chimeric gene was then amplified by using Pfu and cloned into the blunt-ended Sma I binary vector pBAR-35S (Accession no. AJ251014, gift from Zs. SchwarzSommer, Max Planck Institute, Cologne, Germany), carrying a CaMV35S promoter/terminator. Independent clones were sequenced to detect any mutations due to PCR. This construct and a control containing the EGFP sequence were used for transformation.

Agrobacterium tumefaciens-mediated transient expression, drug and heat shock treatments

Transient expression in tobacco and Arabidopsis leaves was performed essentially as described by Batoko et al. (2000) using A. tumefaciens strain GV3101 bearing the appropriate binary vector. Transformed plants were then incubated under normal growth conditions as described above and leaf fragments were mounted in water $48-72 \mathrm{~h}$ after Agrobacterium infiltration and analysed using laser scanning microscopy. The level of atRSp31GFP expression under the control of the CaMV35S promoter was remarkably stable and constant among transformed cells. Fragments of transformed leaves were used for drug treatments. Briefly, leaf tissue was submerged in, or floated on, $5 \mu \mathrm{M} \alpha$-amanitin 
(Sigma), $5 \mu \mathrm{M}$ okadaic acid (Calbiochem), $5 \mu \mathrm{M}$ staurosporine (Sigma) or water as a control for different times (1, 3, 5 and $7 \mathrm{~h}$ ). For heat-shock experiments, plants were placed in a culture chamber for $1 \mathrm{~h}$ at $42^{\circ} \mathrm{C}$ and leaf fragments were processed for confocal imaging. Plants were then put back into the original growth chamber.

Plant transformation and selection

For transformation, Arabidopsis plants were routinely grown in a culture chamber under short-day conditions $(8 \mathrm{~h}$ light/16 h darkphotoperiod) for 8-9 weeks and were then transferred to long-day conditions. The in planta vacuum infiltration method was performed to obtain germinal transformants (Bechtold and Pelletier1998). After transformation, plants were grown under the same conditions as prior to transformation. At least 20 plants were transformed and seeds were collected from individual plants. T1 transformants were obtained by selecting seeds for resistance to glufosinate-ammonium (50 $\mu \mathrm{g} / \mathrm{ml})$ (Riedel-de-Haën) on sterile plates. After 10 days of growth, glufosinate-resistant plants were removed from plates to be processed for observation of fluorescence or planted directly into soil at the two-leafstage to obtain T2 and $\mathrm{T} 3$ progeny.

Whole-mount in situ hybridization and fluorescence immunostaining

Plasmid construction, preparation of U1 and U6 probes and wholemount in situ hybridization were performed as described previously (Beven et al.1995; Boudonck et al.1998). Controls for in situ hybridization were performed using sense riboprobes or RNasetreated $(10 \mathrm{mg} / \mathrm{ml}$ RNase A) samples. The spliceosomal factors $\mathrm{U}^{2} \mathrm{~B}^{\prime \prime}$ and atRSp31 were immunodetected using the mouse monoclonal 4G3 (Euro-diagnostica) and rabbit protein A purified IgG or affinity purified antibodies (Burke et al.1982) anti-atRSp31 at a 1:100 dilution, respectively. For snRNA labelling, formaldehyde-fixed samples were embedded in paraffin by standard procedures and $5 \mu \mathrm{m}$ deparaffined sections were incubated with the primary mouse monoclonal trimethylguanosine cap antibodies (m3G, Euro-diagnostica). Secondary anti-mouse or anti-rabbit antibodies conjugated to fluorescein isothiocyanate (FITC) or AlexaFluor 546 (Molecular Probes) were used. As a control for immunofluorescence, samples were incubated in the presence of either the pre-immune serum or the secondary antibody only. Nuclei were stained with $50 \mathrm{ng} / \mathrm{ml} \mathrm{4}$,6-diamidino-2-phenylindole. Samples were mounted with an antifade medium and observed with the confocal microscope.

\section{Confocal imaging}

The spatial distribution of immunolabelling and GFP fluorescence were examined with a Leica TCS SP2 inverted confocal laser microscope (Leica Microsystems, Germany) equipped with argon and two helium-neon lasers, and an acousto-optical tunable filter (AOTF) for excitation intensity. Digitized images were acquired using a $63 \times \mathrm{NA} 1.5$ Plan-Apo water-immersion objective at $1024 \times 1024$ pixel resolution. The diameter of the pinhole was always set up equal to the Airy unit. For each nucleus, serial optical sections were recorded with a Z-step of $\sim 0.2 \mu \mathrm{m}$. Images were acquired under identical conditions and we ensured that the maximal fluorescence signal was not saturating the PMTs. For four-dimensional imaging, a XYZ multi-image series was defined by $\mathrm{Z}$ sectioning and $\mathrm{Z}$ series were then acquired manually every $10 \mathrm{~min}$ for $90 \mathrm{~min}$. Series of optical sections were either projected as average/maximal projections or as stereo-pair using Leica software (version 2.5). Captured images were exported as TIFF format files and further processed using Adobe Photoshop.
Electron and immunoelectron microscopy

For EM, seeds and root fragments were fixed in $2 \%$ glutaraldehyde in phosphate buffer, $\mathrm{pH} 7.4$ and subsequently processed for Epon embedding. Ultrathin sections were observed with a Zeiss EM900 electron microscope. For the three-dimensional (3D) reconstructions of Arabidopsis nuclei, serial ultrathin sections were collected on single-slot grids. For quantitative analysis of nuclear bodies, at least three meristems per germination time were evaluated and, per meristem, 160 nuclear sections and 100 nucleolar sections were randomly observed for counting the dense bodies (DBs) and CBs, respectively. The minimal number of sections to be considered was determined for each time point by counting the nuclear bodies in an increasing number of sections until their mean number became stable. The distributions of the numbers of nuclear bodies were compared by the Kruskal-Wallis non-parametric method. All statistical data were processed using Statistica 6.0 (Statsoft). Preembedding immunodetection was performed as follow: seeds and root meristems were fixed in $4 \%$ formaldehyde, washed in PEM, $0.2 \%$ NP40 (50 mM PIPES, KOH, $\mathrm{pH}$ 6.9; 5 mM EGTA; $5 \mathrm{mM}$ $\mathrm{MgSO}_{4}$.) and subsequently digested for $2 \mathrm{~h}$ with driselase $(1 \%)$ cellulase $(0.5 \%)$, pectolyase $(0.025 \%)$. After washing in PEM, $0.2 \%$ NP40, samples were treated with DMSO (0.5\%). Cryosections approximately $90 \mu \mathrm{m}$ thick were made and allowed for a blocking step in PEM, $0.2 \%$ NP40, 3\% BSA. Sections were successively incubated at $4{ }^{\circ} \mathrm{C}$ overnight with mouse monoclonal 4G3 antibodies at a dilution of 1:100 and anti-mouse antibodies conjugated to $10 \mathrm{~nm}$ gold particles at a 1:100 dilution. Samples were then embedded in Epon for EM observation.

\section{Results}

\section{Nuclear bodies are observed by EM}

The ultrastructure of maize and Arabidopsis root nuclei has been observed at different time points during germination and early seedling development. Comparison of nuclear architecture did not reveal gross structural differences between maize and Arabidopsis. Several aspects of nuclear ultrastructural changes during germination of maize and Sinapis alba have previously been described in detail, except for the nuclear bodies on which we focused our present observations (Deltour et al.1986). At each germination time, nuclear bodies were observed in both species (Fig. 1 and not shown). Cajal bodies exhibited a fibrillar texture and were localized either inside a slight depression on the surface of the nucleolus or free in the nucleoplasm, referred to, respectively, as nucleolus-associated CBs (NCBs) and free CBs (FCBs) (Fig. 1). Up to $72 \mathrm{~h}$ of germination, CBs appeared mainly associated with the nucleolus while after this period they were most often free within the nucleoplasm (Figs. 1, 2). Both their morphology and localization appeared identical between species.

In maize, $\mathrm{DBs}$ were easily distinguishable from $\mathrm{CBs}$ by their structure and smaller diameter. They were very rare in quiescent nuclei but their number progressively increased during germination with the resumption of nuclear activity (Fig. 1). Most often, they have been observed free in the nucleoplasm but were sometimes observed in close association with the nucleolus (Fig. 1). They displayed a dense fibrillar structure forming a compact spherical body, sometimes showing a crown of 
Fig. 1 A-D Electron micrographs of cortical root cells of Zea mays at different time points during germination. In quiescent cells ( $\mathbf{A}$ ) and at $8 \mathrm{~h}$ after imbibition ( B) crescentshaped nucleolus-associated Cajal bodies (NCBs, arrows) are located at the nucleolar periphery. After $24 \mathrm{~h}$ of germination ( $\mathbf{C}$ ), CBs become more spherical and after $120 \mathrm{~h}$ (D), they appear in the nucleoplasm as well (free $\mathrm{CBs}$, FCBs). High magnification of one $\mathrm{CB}(\mathbf{E})$ and of dense bodies (DBs, F). G EDTA-regressive staining of a fully active maize nucleus showing dispersed granules between and highly contrasted fibrils around bleached chromatin clumps. H Frequency distributions of the numbers of total CBs, NCBs, FCBs and DBs. Different letters on the tops of columns indicate statistical differences at $P<0.05$. Bars represent $1 \mu \mathrm{m}$ ( A-D); $200 \mathrm{~nm}(\mathbf{E}, \mathbf{F}) ; 500 \mathrm{~nm}(\mathbf{G})$
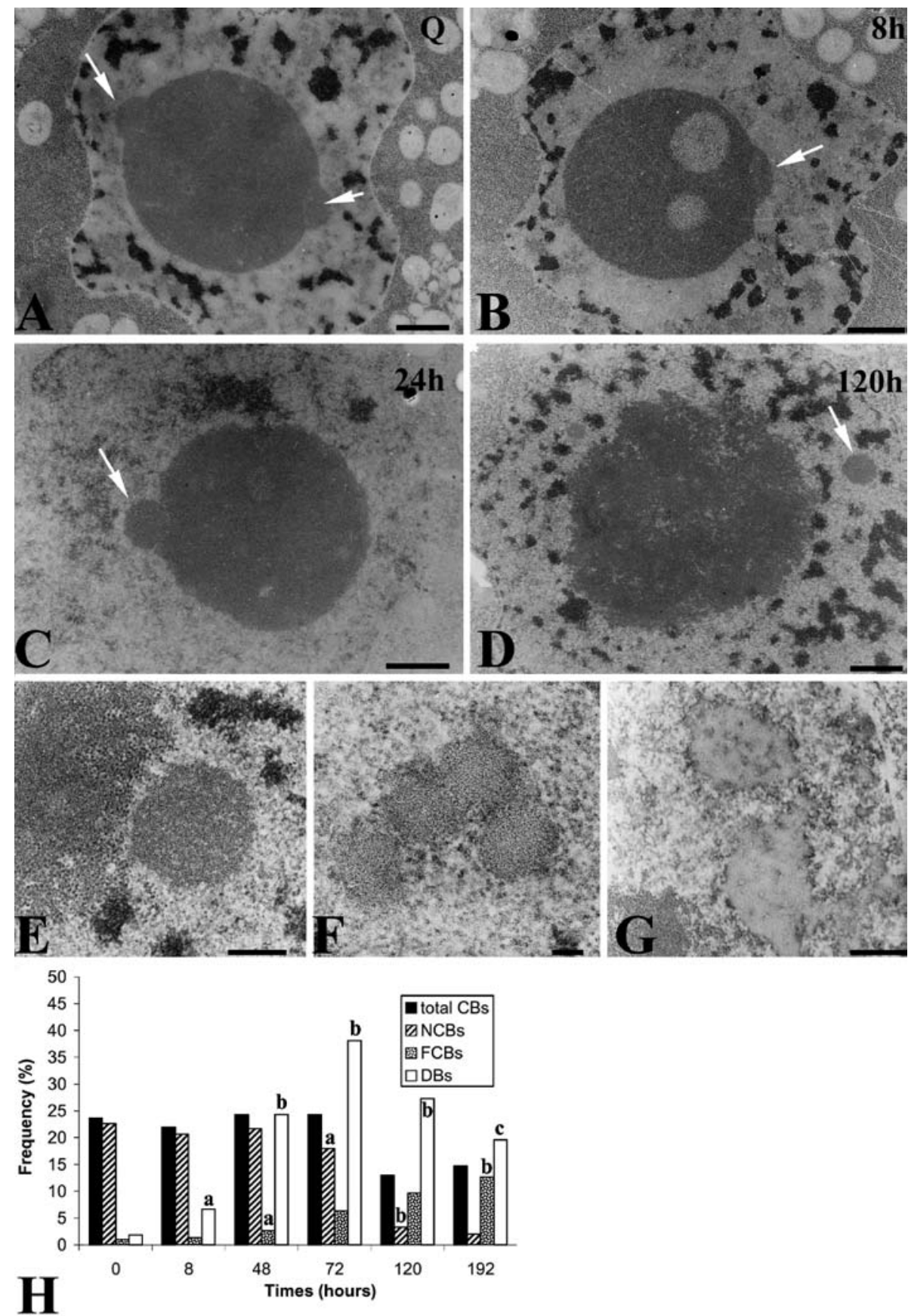

dense material. Although often seen as single, these bodies were occasionally seen in groups of two or three (Fig. 1F). No dense bodies were observed in Arabidopsis as confirmed by our 3D reconstruction of nuclei (not shown). Their absence in Arabidopsis at any germination time could suggest species-specific functions. Further studies will be needed to identify specific molecules located in DBs and to gain insight into their possible role in nuclear processes. The ribonucleoprotein nature of $\mathrm{CBs}$ and DBs was strongly suggested by the persistence of their contrast after an EDTA-regressive staining technique that revealed ribonucleoprotein material around bleached chromatin clumps and individual or small groups of granules in the interchromatin region (Fig. 1G).

Nuclear body numbers were analysed in maize meristematic cortical cells of the primary root during germination using EM (Fig. 1H). No important fluctuation of total CBs per nucleus was observed during the first $72 \mathrm{~h}$. However, discrimination between FCBs and NCBs showed a slight increase and decrease, respectively, in their number. These variations were further accentuated after this germination time. Interestingly, the significant decrease of $\mathrm{CB}$ frequency was the direct consequence of the decrease in NCB frequency while the FCB frequency significantly increased. The frequency of DBs increased 
Fig. 2 A Distribution of U2B ${ }^{\prime \prime}$ and of $\mathrm{m} 3 \mathrm{G}$ snRNAs in nuclei from maize root tip meristems at different time points during germination. The antigens are localized as a weak punctate nucleoplasmic labelling in quiescent nuclei ( $Q$ ) and display a more homogeneous pattern with their concentration in bright spots corresponding to $\mathrm{CBs}$ with the onset of germination. Bars represent $8 \mu \mathrm{m}$. B U2B" labelling of the CBs at 8 and $120 \mathrm{~h}$ of germination, respectively, visualized by electron microscopy. Bars represent $200 \mathrm{~nm}$. C In situ localization of U1 and U6 snRNAs in quiescent $(Q)$ and active nuclei. Bars represent $8 \mu \mathrm{m}$. D Immunoblot analysis of bacterially expressed proteins $(1,2)$ and of total proteins prepared from Arabidopsis inflorescences $(4,5)$ with anti-atRSp31 antibodies $(1,4)$ or pre-immune serum $(2$, 5). 3 Coomassie blue-stained gel shows the overall protein composition from inflorescences. E Distribution of atRSp31 in nuclei from

Arabidopsis root tip meristems during germination and in trichoblast ( tricho.). Bars represent $4 \mu \mathrm{m}$
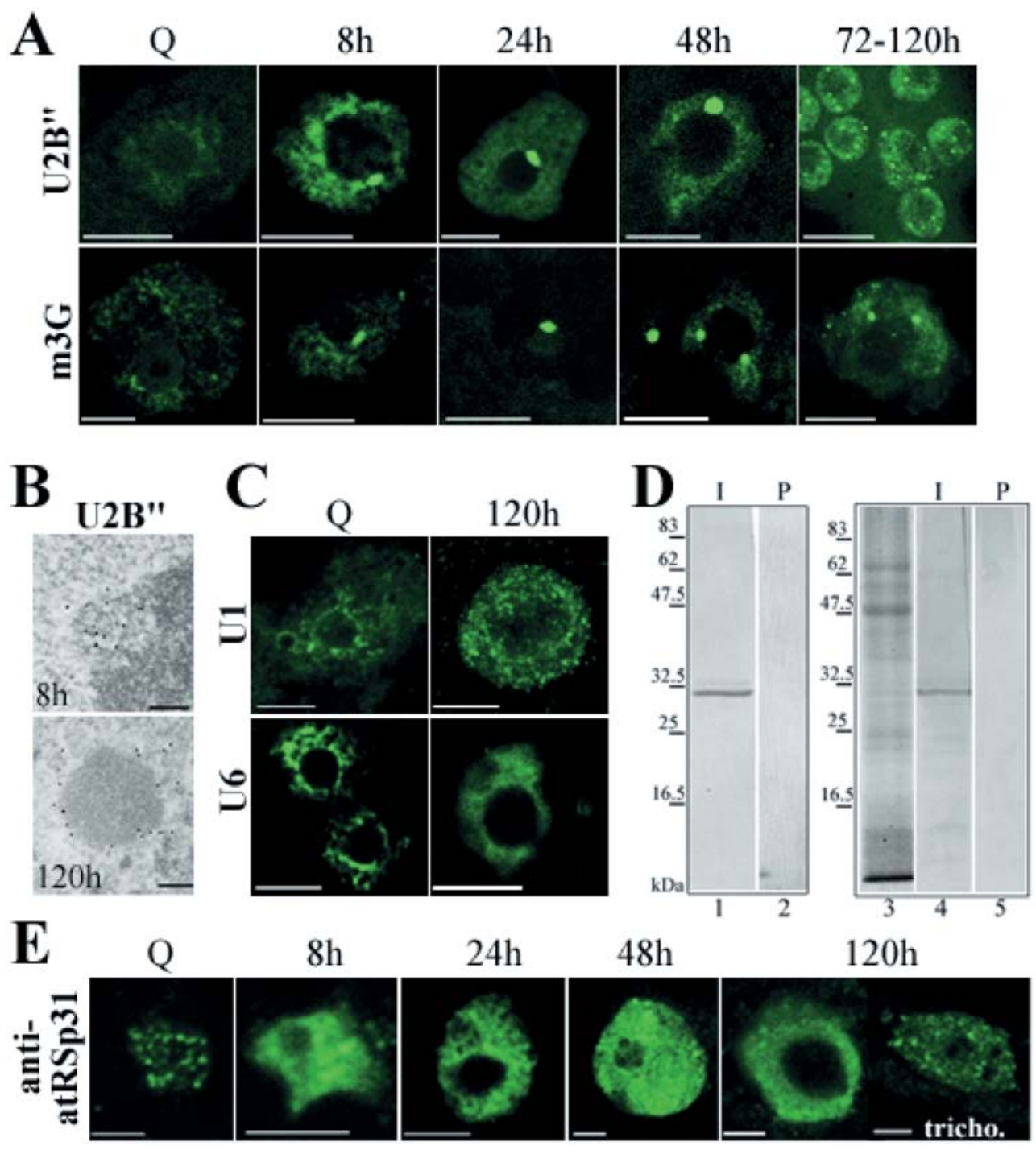

$48 \mathrm{~h}$

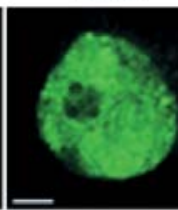

$120 \mathrm{~h}$

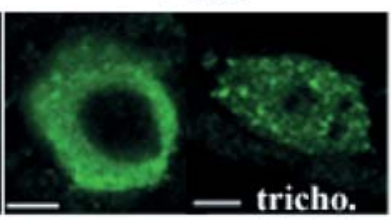

by 20 -fold during the first $72 \mathrm{~h}$ of germination (from 1.9 to $38.1 \%$ ). The results of the statistical analysis are shown in Fig. 1.

\section{Splicing factor localization during germination}

As U2B" protein is a good marker of CBs in plants (Beven et al.1995; Boudonck et al.1998,1999; Acevedo et al.2002; Cui and Moreno Diaz De La Espina2003), its distribution was analysed by immunocytochemistry on both maize and Arabidopsis root meristematic cells by confocal microscopy. The labelling appeared very similar, if not identical, and Fig. 2A shows only the results of U2B" staining in maize nuclei due to space limitation. In quiescent cells, U2B" localized as weak punctate nucleoplasmic labelling and a confocal Z-series through nuclei showed that CBs observed by EM were not labelled. At $8 \mathrm{~h}$, interestingly, U2B" showed a heterogeneous nucleoplasmic distribution and became highly enriched in pre- existing CBs in both species as confirmed by immunoelectron microscopy (Fig. 2B). This latter technique also showed a weak density of particles in the nucleoplasm and no labelling over the nucleolus, cytoplasm or DBs (not shown). Later, U2B" was dispersed more homogeneously throughout the nucleoplasm and concentrated in at least one fluorescent spot per nucleus (Fig. 2A). The foci associated with the nucleolus were generally larger than the free ones.

The distribution of snRNAs observed using anti- $\mathrm{m}_{3} \mathrm{G}$ snRNA antibodies was similar to the U2B" protein distribution in both species (Fig. 2A and not shown). One slight difference was a labelling of the CBs in quiescent nuclei. SnRNAs being among the best-characterized plant splicing factors, genes encoding U1 and U6 have been cloned and in situ hybridization was performed with anti-sense RNA probes during germination. Again, U1 and U6 probes showed similar distribution patterns in both maize and Arabidopsis. U1 and U6 could be detected in quiescent nuclei as shown in Fig. 2C. In active cells, 
the strongest nuclear signal was localized as bright small foci for U1 and a more homogeneous bright interchromatin network for U6. No labelling of nuclear bodies was observed in quiescent and active nuclei. In different controls for immunocytochemical protocols and in situ hybridization, no labelling was seen over nuclei (not shown).

To investigate the subcellular distribution of a plant SR splicing factor, rabbit polyclonal antibodies were raised against atRSp31, which is characterized by the presence of one N-terminal RRM and a C-terminal RS domain (Lopato et al.1996). The specificity of the affinitypurified antibodies was tested by immunoblot analysis on a protein extract from E. coli and from Arabidopsis plants (Fig. 2D). It showed that immune rabbit serum raised against recombinant atRSp31 specifically recognized His-atRSp31 in the crude extract of E. coli (Fig. 2D). With the protein extract from Arabidopsis plants, this antibody stained a single band at $31 \mathrm{kDa}$, corresponding to the expected molecular mass of fulllength atRSp31. The pre-immune serum from the same rabbits gave no signal (Fig. 2D). Thus, our polyclonal antibody is highly specific to the Arabidopsis atRSp31 factor. Using these antibodies in immunofluorescence confocal microscopy, we analysed the subcellular distribution of atRSp31 in meristematic cortical root cells during the early germination of $A$. thaliana (Fig. 2E). AtRSp31 was detected as punctate foci in quiescent nuclei. With the onset of nuclear reactivation as early as $8 \mathrm{~h}$ after seed imbibition, the foci organization of atRSp31 became less apparent and a more homogeneous distribution was observed in cortical cells of the root meristem (Fig. 2E). However, a speckled distribution of atRSp31 was observed in the nucleoplasm of trichoblasts and atrichoblasts in the elongation and differentiation zones (Fig. 2E). Our anti-atRSp31 sera were tested on maize embryos but no labelling was detected (not shown), showing the high specificity of the anti-atRSp31 antibodies.

\section{RT-PCR analyses during germination}

The overall modifications in the distribution of splicing factors during early germination suggest that the splicing process could slowly and/or sequentially resume. In order to verify such a possible sequential resumption, we looked for intron-containing genes in databases that could be expressed as early as seed imbibition and determined their transcript profile during early germination. We performed RT-PCR analysis with terminal cDNA primers of actin, HAT and cyclin on total RNA extracted from maize root tips. As shown in Fig. 3, PCR of actin yielded two bands corresponding to the sizes of the unspliced premRNA and spliced mRNA (2.201 and $1.128 \mathrm{~kb}$, respectively); PCR of cyclin and HAT yielded single bands of $1.437 \mathrm{~kb}$ and $1.404 \mathrm{~kb}$, respectively, corresponding to the sizes of spliced mRNAs. RT-PCR was carried out on different maize varieties and the only difference was the

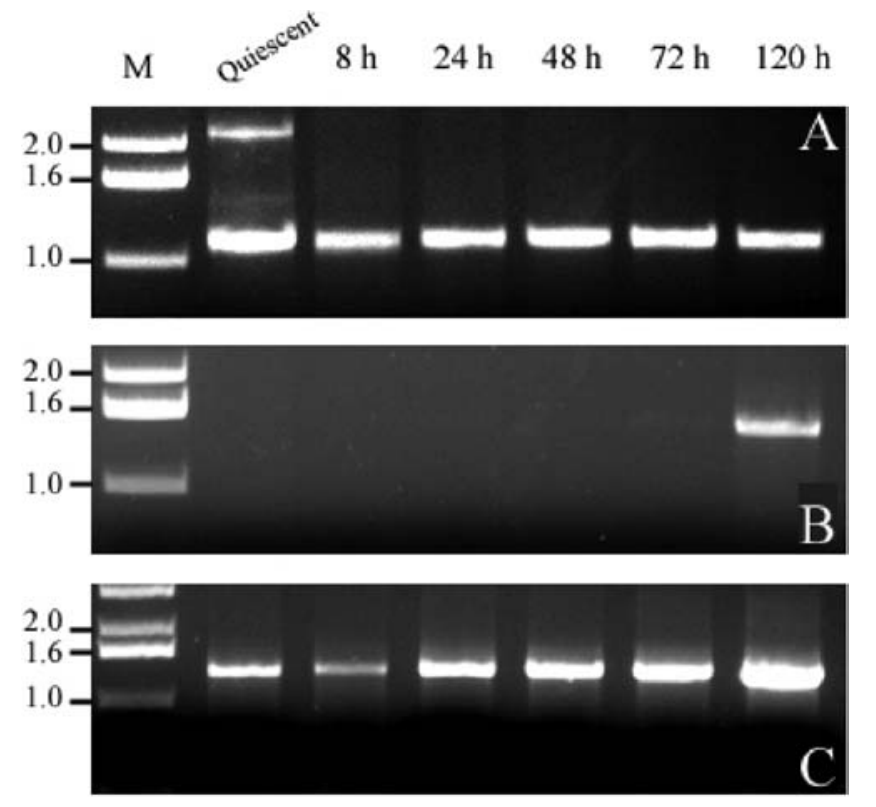

Fig. 3 Transcript analysis of the expression of actin (A), HAT ( B) and cyclin ( $\mathbf{C}$ ) during early germination of Zea mays. Ethidium bromide-stained gels showing products of the reverse-transcribed polymerase chain reaction (RT-PCR). Lanes 2-7 Products from maize RNA amplified at different time points of germination. Units are in kilobases. Lane $M$ markers

PCR products for HAT and actin appearing as single bands of correctly spliced mRNAs in quiescent embryos (not shown). The observation that unspliced pre-mRNAs and spliced mRNAs coexist in quiescent embryos suggests that transcription and splicing could be dissociable processes in some physiological situations.

Localization of atRSp31 after transient expression, drug treatment and heat shock

The experiments described so far examined the spatial localization of splicing factors by imunocytochemistry on fixed tissues, which did not assess the dynamics of any factor. To examine further the subcellular localization and dynamics of atRSp31 in living plant cells, we generated a translational fusion construct of this splicing factor with GFP linked to the C-terminus of atRSp31 under the control of the CaMV35S promoter. First, we used transient expression to develop a rapid and efficient assay for the in vivo spatial organization of plant SR splicing factors. Fluorescence of GFP-tagged atRSp31 was observed in Arabidopsis and tobacco leaf cells after agroinfiltration-based transient expression. We ensured that agroinfiltration and subsequent fluorescent analysis did not induce cell death or necrosis. Moreover, we did not observe any toxic effects of atRSp31-GFP in transiently overexpressing cells. The Agrobacterium inoculation only induced weak chlorosis in tobacco, which facilitated the identification of the transiently expressing cells. The fluorescence of GFP could be easily 
Fig. 4A-C Agrobacterium mediated transient expression of atRSp31-GFP (green fluorescent protein) fusions or free GFP under the control of the CaMV 35S promoter. A Left panels: low-magnification confocal microscopy through epidermal cells expressing atRSp31-GFP and free GFP merged with chlorophyll autofluoresence ( bars represent $80 \mu \mathrm{m})$. Middle and right panels: high resolution confocal microscopic sections show discrete nuclear speckles in tobacco and Arabidopsis leaf cells expressing atRSp31-GFP ( bars represent $4 \mu \mathrm{m}$ ). Relative intensity fluorescence profiles are plotted along the line across the nucleus. B Selection of serial optical sections through the nucleus of an atRSp31-GFP expressing Arabidopsis leaf cell. In addition to diffuse fluorescence, speckle-like structures are apparent throughout the nucleus. Bar represents $2 \mu \mathrm{m}$. C AtRSp31-GFP localization after drug treatments $(\alpha$-amanitin, okadaic acid and staurosporine) and heat shock. ( $\mathrm{Nu}$ nucleolus.) Bar represents $2 \mu \mathrm{m}$

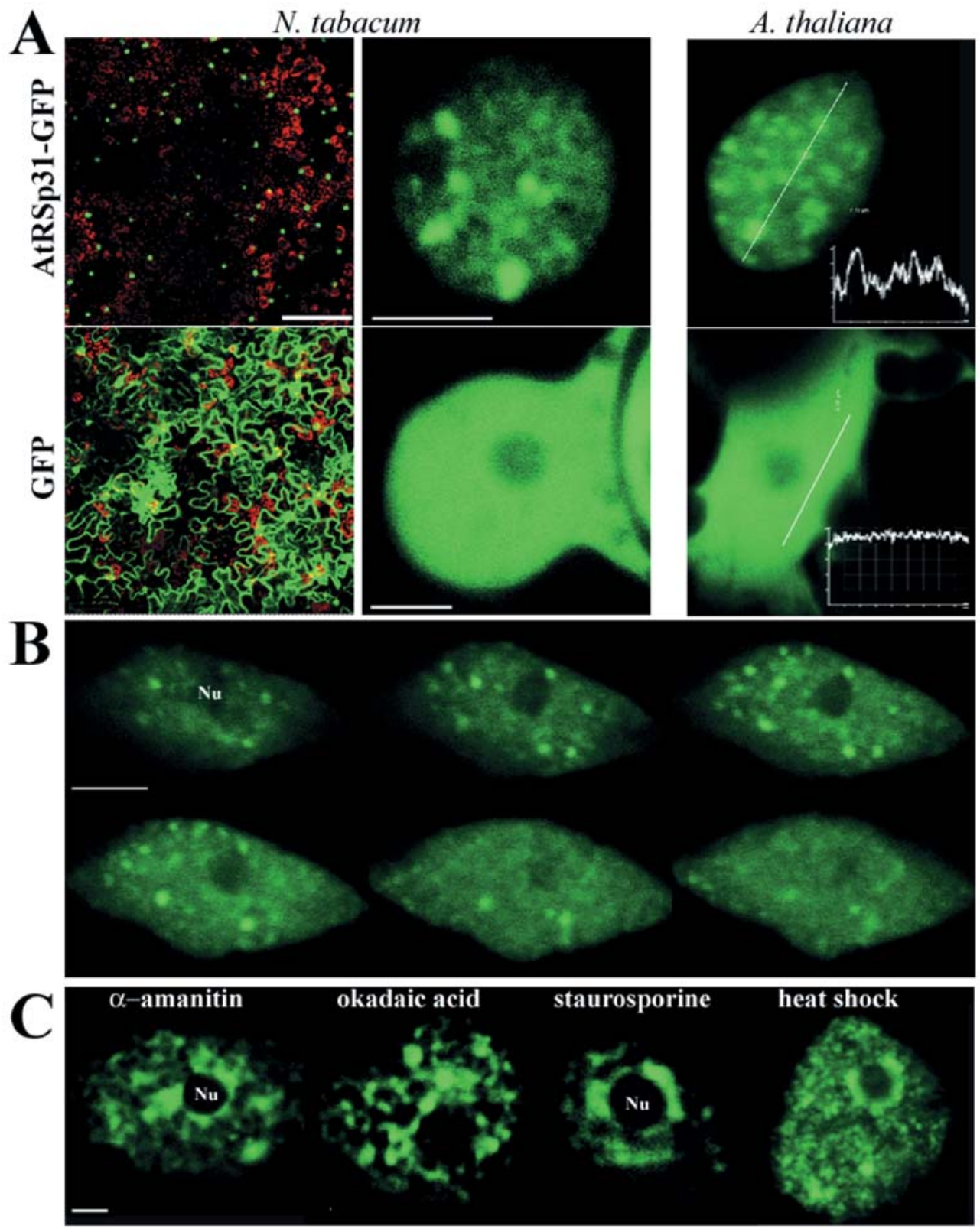

detected by epi-fluorescence using an FITC filter block. Many cells were observed to express the fluorescent proteins as early as $24 \mathrm{~h}$ after infiltration. The expression level reached an optimum after $48 \mathrm{~h}$ and the fluorescence was very stable and could still be detected for at least 56 days in both species although the efficiency of the method was always higher in tobacco (not shown). As previously visualized using anti-atRSp31 antibodies and indirect immunofluorescence, the speckled pattern of atRSp31-GFP was clearly apparent in both tobacco and Arabidopsis leaf cells (Fig. 4). To our knowledge, this is the first report of the efficient transient transformation of Arabidopsis leaves after Agrobacterium inoculation. The spatial distribution of free GFP was observed by expressing a binary vector containing only the GFP gene downstream of the CaMV35S promoter. As expected from published data, expression of free GFP produced high and homogeneous fluorescence in both cytoplasm and nucleus due to passive diffusion through nuclear pores (Fig. 4A). No speckled accumulation of GFP in either cytoplasm or nucleus was observed (Fig. 4). Confocal Z-series of tobacco and Arabidopsis nuclei clearly demonstrated a speckled fluorescence organization of atRSp31-GFP in the entire nuclear depth (Fig. 4B). The analysis of GFP fluorescence at different time points after agroinfiltration showed that nuclear speckles appeared as early as fluorescence could be detected $(\sim 24 \mathrm{~h})$, suggesting that subnuclear distribution of atRSp31-GFP is relatively independent of its overexpression and overaccumulation.

Inhibition of RNA polymerase II transcription by $\alpha$ amanitin caused the disappearance of the diffuse fraction 
Fig. 5 A-E Confocal microscopic analysis of a transgenic seedling expressing atRSp31GFP. Bright-field image ( A) and in vivo analysis of GFP fluorescence ( B) of a primary root expressing the fusion protein. The maximal projection shows the nuclear localization of atRSp31-GFP in every cell of the root. High resolution images of nuclei from one meristematic cell ( C), one atrichoblast cell of the elongation zone ( D) and one trichoblast cell of the differentiation zone ( $\mathbf{E})$. F-H Fixed-trichoblast cell expressing atRSp31-GFP ( green) immunostained with antibodies against U2B" ( red). The overlap ( $\mathbf{H})$ shows distinct nuclear localization of the speckles and Cajal body ( arrow). I-L Ultrastructure of nuclei of wildtype meristematic (I) and trichoblast $(\mathrm{J})$ cells and of transgenic meristematic $(\mathrm{K})$ and trichoblast (L) cells. In trichoblast cells, some microclusters of interchromatin granules are observed (see insert for high magnification). Bars represent $200 \mu \mathrm{m}(\mathbf{A}, \mathbf{B}) ; 1 \mu \mathrm{m}(\mathbf{C}-\mathbf{L})$
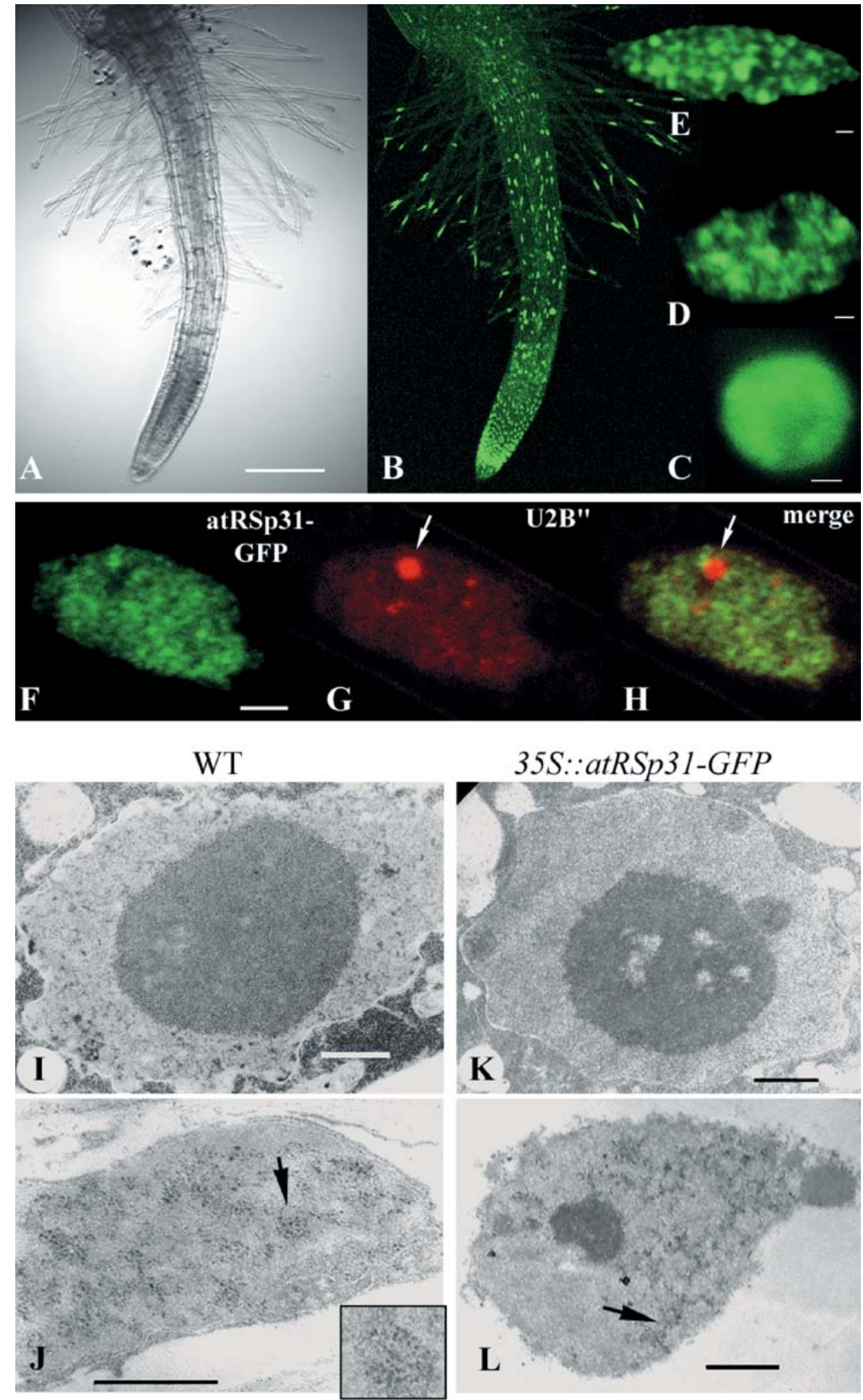

of atRSp31-GFP and its redistribution into larger nuclear domains (Fig. 4C). The use of okadaic acid and staurosporine (Ser Thr phosphatase and kinase inhibitors, respectively) on transformed leaves provoked a similar disappearance of the diffuse atRSp31-GFP and its con- centration into bigger speckles and around the nucleolus (Fig. 4C). Heat shock treatment, known to disrupt spliceosomal components, led to accumulation of atRSp31-GFP around the nucleolus as well (Fig. 4C). 
Fig. 6 Four-dimensional imaging of an atrichoblast nucleus showing movement, fusion and budding of atRSp31-GFP over time ( arrows). $\mathrm{Z}$ series were acquired at $\sim 10$ min intervals and three-dimensional stereopair images were reconstructed for each time point. The time in minutes is indicated at the top of each section. Bar represents $2 \mu \mathrm{m}$

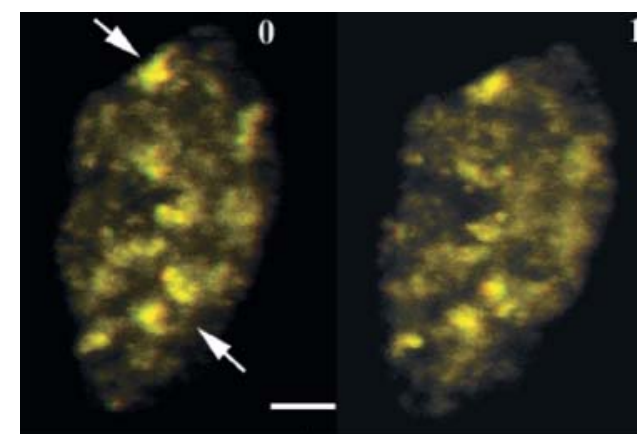

\section{0}

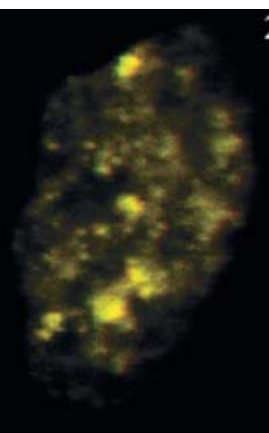

20

30

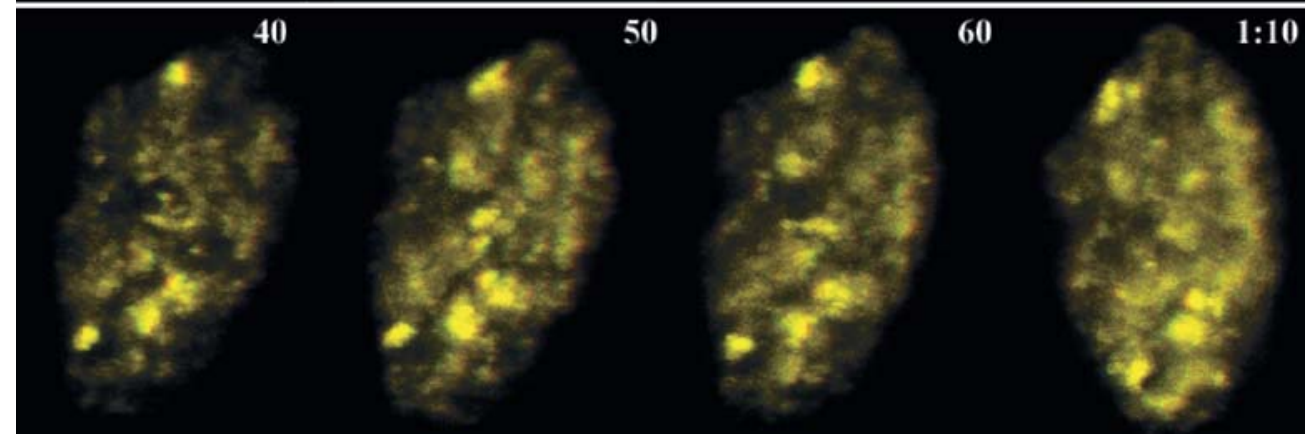

Dynamic nuclear localization of atRSp31 in Arabidopsis

Since a speckled organization of the atRSp31 SR splicing factor occurred after our transient expression experiments (strongly suggesting a functional localization of the fusion protein), we generated transgenic Arabidopsis plants stably expressing the atRSp31::GFP fusion construct, allowing us to compare different cell types and developmental stages. Similar results were obtained with many independent lines (not shown). A low-magnification fluorescence analysis showed a clear nuclear localization of the fusion protein in the whole seedling root (Fig. 5). As already reported (Chytilova et al.2000), nuclei within root-hair cells were observed to have a complex nonspherical shape. In meristematic cells, uniform fluorescence was observed with no apparent accumulation of atRSp31-GFP in foci (Fig. 5C). The most impressive speckled distribution of GFP-tagged atRSp31 could be observed in epidermal cells (both atrichoblasts and trichoblasts), similar to what we observed using antibodies (Figs. 5, 6). Interestingly, the analysis of transgenics and of transient transformation demonstrated that overexpression of atRSp31-GFP resulted in its accumulation in speckles in a particular cell or tissue type. We next sought to observe whether overexpressed atRSp31-GFP accumulated in CBs. U2B" immunostaining in atRSp31GFP-expressing cells displayed a diffuse U2B" distribution, with additional bright CBs. The nucleoplasmic diffuse labelling of $\mathrm{U}^{2} \mathrm{~B}^{\prime \prime}$ and atRSp31-GFP colocalized but $\mathrm{U}^{2} \mathrm{~B}^{\prime \prime}$ protein did not accumulate in the speckles (Fig. 5F-H). Next, we legitimately wondered whether the overexpression of atRSp31-GFP under the control of CaMV35S promoter could modify the organization of nuclei. In other words, could overexpressed atRSp31-GFP induce the development/formation of IGCs in plant cells? High-resolution nuclear organization of Arabidopsis transgenics was investigated by EM and compared with that of wild-type seedlings. Observations were made in both meristematic and differentiation zones of the root. No gross changes in overall nuclear ultrastructure were observed between T2/T3 transgenics and wild-type, either in meristematic cells or in the differentiation zone (Fig. 5I-L). Although smaller than in mammalian cells, micro-clusters of IGs were nevertheless observed in trichoblasts (Fig. 5J, L).

Since the dynamic properties of speckles in plant cells are largely unknown, high-resolution 3D time-lapse imaging of atRSp31-GFP in T2 and T3 seedlings was performed (Fig. 6). Four-dimensional analysis of meristematic nuclei did not show any modification in the fluorescence pattern over a long period (P. Motte, unpublished data). However, in atrichoblasts, the stereo images presented in Fig. 6 show complex movement and shape modification of atRSp31-GFP over a long period. Although the nuclear speckles of atRSp31-GFP remain in relatively stable spatial domains during a time-lapse analysis of more than $90 \mathrm{~min}$, their periphery could display a likely non-random budding and fusing. Rapid movement (a few seconds) of atRSp31-GFP was also visible at the periphery of the speckles (P. Motte, unpublished data). Unfortunately, four-dimensional analysis of root-hair nuclei was difficult to perform because of the rapid and constant movements of nuclei along the cells during laser scanning (P. Motte, unpublished data). 


\section{Discussion}

\section{Nuclear bodies}

The nucleus is now recognized as a complex organelle with spatially organized territories generically referred to as nuclear bodies. With a diameter ranging from less than $1 \mu \mathrm{m}$ to more than $10 \mu \mathrm{m}, \mathrm{CBs}$ have been identified in both animal and plant cells and appear as highly dynamic structures (Beven et al.1995; Boudonck et al.1998,1999; Jennane et al.1999; Matera1999; Lewis and Tollervey2000). In this work, CBs were observed in quiescent and germinating cells in both species. In quiescent nuclei, $\mathrm{CBs}$ are very often in close association with the nucleolus and they do not label with anti-U2B" antibodies or U1/U6 antisense probes. This suggests either that splicing factors are not strictly required for the assembly of $\mathrm{CBs}$ or that splicing factors can leave CBs (as a result of seed desiccation) without affecting their morphology. Soon after seed imbibition, pre-existing CBs became highly enriched in U2B" . Their content modification correlates with the resumption of nuclear reactivation during early germination. At $72 \mathrm{~h}$ of germination, a significant decrease of total $\mathrm{CBs}$ with a concomitant decrease of NCBs and an increase of FCBs were observed. Similar variations in the number and localization of $\mathrm{CBs}$ from dormant/quiescent to transcriptionally active cells have been reported in other plants (Beven et al.1995; Boudonck et al.1998,1999; Jennane et al.1999; Acevedo et al.2002; Cui and Moreno Diaz De La Espina2003). In maize, the decrease of $\mathrm{CBs}$ can be related to the resumption of mitotic activity in the meristem. Indeed, previous studies have established that the majority of the cells are in G1 and that the first wave of mitosis occurs at about $72 \mathrm{~h}$ after imbibition (Deltour1985). This is in good agreement with the results of Boudonck et al. (1999) who have analysed the localization of GFP-tagged U2B" protein in BY2 tobacco cells and Arabidopsis transgenics. In living plant cells they have observed movements of CBs from the nuclear periphery to the nucleolus, their frequent fusion and a decrease in CBs per nucleus directly after cell division (Boudonck et al.1999).

Interestingly, we detected splicing factors in inactive quiescent nuclei. These factors were enriched in particular ribonucleoprotein domains, as also described in dormant onion cells and named micro-speckles (Cui and Moreno Diaz De La Espina2003). As suggested by Cui and Moreno Diaz de la Espina (2003), quiescent microspeckles could be storage sites of splicing factors, becoming biologically active and redistributed with the onset of nuclear activation. Although few data are available on both the nature and the localization of stored mRNAs in the quiescent embryos, these quiescent/ dormant micro-speckles could alternatively represent residual preformed mRNA linked to processing factors that have survived the desiccation programme occurring at the end of seed maturation. Although we do not know whether polyadenylated RNAs are located within these micro-speckles, it has been reported that inhibition of mammalian RNA polymerase II induced a population of stable long-lived poly(A) RNA associated with premRNA splicing factors forming IGCs (Huang et al.1994). Similarly, seeds becoming metabolically quiescent are characterized by transcription that is slowly arrested and preformed mRNAs could remain linked to processing factors in quiescent nuclei. Our RT-PCR data suggest that quiescent long-lived mRNAs are correctly spliced mRNAs, regardless of where these are located.

Speckles, or not speckles, in the plant nucleus: the beginning of an answer?

The compartmentalization of essential SR splicing factors has been extensively studied in animal cells (Lamond and Earnshaw1998; Eils et al.2000; Misteli 2000 Allemand et al.2001; Dundr and Misteli2001). SR proteins concentrate in precise subnuclear speckles consisting of many irregularly shaped domains in the interchromatin compartment. In mammalian cells, speckles correspond to IGCs composed of particles measuring $20-25 \mathrm{~nm}$ in diameter and perichromatin fibrils. Whereas a plethora of Arabidopsis SR splicing factors has been identified (Lorkovic and Barta2002), their precise subcellular distribution is largely unresolved. Since the Arabidopsis atRSp31 protein has been shown to complement the HeLa S100 extract (Lopato et al.1996), there was significant interest in investigating its localization and dynamic redistribution within the context of plant nuclear organization. Consistent with its role as a splicing factor (Lopato et al.1996,2002), atRSp31 protein was exclusively localized in the nucleus by indirect immunofluorescence. Moreover, atRSp31 factors displayed a heterogeneous speckled distribution in root epidermis cells (trichoblasts and atrichoblasts). The restricted nuclear localization of atRSp31, its diffuse distribution and accumulation in a number of discrete speckles were further observed by transient and stable expression of GFP-tagged atRSp31 in both tobacco and Arabidopsis cells.

Although atRSp31 can display a speckled organization, the presence of IGCs in plant cells is still a matter of debate (Testillano et al.1993; Acevedo et al.2002; Cui and Moreno Diaz De La Espina2003). A clear IGC organization such as the one described in mammalian cells has never been described in plant cells by EM although in some cell types, some small clusters of particles of 20 $30 \mathrm{~nm}$ can be observed (Testillano et al.1993; Acevedo et al.2002; Cui and Moreno Diaz De La Espina2003; and the present report). Some questions can legitimately be raised as to whether the GFP-tagged atRSp31 is fully functional as an essential splicing factor and whether the speckled conformation of the fusion protein has some biological significance. Due to the strong constitutive CaMV35S promoter, the accumulation of atRSp31-GFP in nuclei could indeed amplify (or create) the speckled organization of SR splicing factors. However, a functional arrangement of atRSp31-GFP rather than an in vivo aggregate artefact due to protein overaccumulation is 
suggested by several arguments. First, in Arabidopsis transgenics (T1, T2 or T3), the most characteristic speckled organization of atRSp31-GFP was observed in the epidermis, where comparable immunofluorescence labelling of the wild-type atRSp31 was detected in fixed root tissues. Second, we did not observe nuclear speckles in root tips (e.g. meristematic zones) where intense fluorescence was nevertheless present, indicating overaccumulation of atRSp31-GFP. Third, our time-lapse and four-dimensional analysis of fusion protein dynamics showed budding movement at, and exchange between, speckle peripheries. Moreover, the redistribution of atRSp31-GFP after heat shock and inhibitor treatments suggests that the fusion protein is functional and that the phosphorylation/dephosphorylation level and the transcriptional activity of the cell regulate its nuclear localization. We have observed that overexpressed mutant atRSp31-GFP proteins with deleted RS domains do not concentrate in discrete subnuclear foci after transient transformation, indicating that the RS domain is essential in directing speckled organization in living plant cells (V. Tillemans and P. Motte, unpublished).

Our report also shows that agroinfiltration-mediated transient expression can provide a fast and powerful tool for the study of the distribution of splicing factors in vivo. Moreover, transient expression in tobacco demonstrates that the presence of endogenous Arabidopsis atRSp31 is dispensable for the speckled organization of atRSp31GFP.

If we clearly show that Arabidopsis SR proteins are localized in nuclear speckled domains, we do not know how (or whether) they fit into the existing model of compartmentalization of mammalian SR splicing factors. We have to consider the possibility that the functions of speckles revealed by atRSp31 have yet to be identified in plants. Interestingly, speckle-like structures have been described in plant nuclei for many developmental regulators as well, like phytochrome or CRY2 photoreceptors (Mas et al. 2000; Kircher et al. 2002; Kevei and Nagy2003). The speckled distribution of these regulatory proteins is suggested to correspond to macromolecular complexes functional in the synthesis and processing of specific target gene transcripts. The speckled organization of plant SR protein might reflect a high and transient demand of splicing factors in response to actively transcribed genes rather than a reservoir of pre-mRNA processing factors shuttling between storage/preassembly sites to transcription sites. We can reasonably ask whether speckles and micro-clusters of IGs observed in Arabidopsis epidermis is possibly linked to a high or low transcriptional activity of these differentiated cells. The speckled distribution of SR proteins in plants could correspond to even larger macromolecular complexes coordinating spatial organization of active euchromatin (from chromatin remodelling to pre-mRNA processing), these hypotheses not being mutually exclusive.

Acknowledgements We are grateful to Dr P. Gérard (Université de Liège; Unité de Recherche de Statistique - Aspects Expérimen- taux) for assistance with statistical analysis, Prof. Marc Boutry (Université Catholique de Louvain, Belgium) for valuable advice with transient transformation and to Prof. Peter Shaw (John Innes Centre, Norwich, UK) for helpful comments. This research was supported by grants from "National Fund for Scientific Research" (grants no. 2.4547.99, 2.4520.02 and 2.4542.00) and from "Fonds Spéciaux du Conseil de la Recherche" of the University of Liège. S.D. was supported by an FRIA grant (Fonds de la Recherche pour l'Industrie et l'Agriculture) and V.T. is supported by FRIA.

\section{References}

Acevedo R, Samaniego R, Moreno Diaz de la Espina S (2002) Coiled bodies in nuclei from plant cells evolving from dormancy to proliferation. Chromosoma 110:559-569

Allemand E, Gattoni R, Bourbon H-M, Stevenin J, Caceres JF, Soret J, Tazi J (2001) Distinctive features of Drosophila alternative splicing factor RS domain: implication for specific phosphorylation, shuttling, and splicing activation. Mol Cell Biol 21:1345-1359

Batoko H, Zheng H-Q, Hawes C, Moore I (2000) A Rab1 GTPase is required for transport between the endoplasmic reticulum and Golgi apparatus and for normal Golgi movement in plants. Plant Cell 12:2201-2218

Bechtold N, Pelletier G (1998) In planta Agrobacterium-mediated transformation of adult Arabidopsis thaliana plants by vacuum infiltration. Methods Mol Biol 82:259-266

Beven AF, Simpson GG, Brown JW, Shaw PJ (1995) The organization of spliceosomal components in the nuclei of higher plants. J Cell Sci 108:509-518

Bewley JD (1997) Seed germination and dormancy. Plant Cell 9:1055-1066

Boudonck K, Dolan L, Shaw PJ (1998) Coiled body numbers in the Arabidopsis root epidermis are regulated by cell type, developmental stage and cell cycle parameters. J Cell Sci 111:36873694

Boudonck K, Dolan L, Shaw PJ (1999) The movement of coiled bodies visualized in living plant cells by the green fluorescent protein. Mol Biol Cell 10:2297-2307

Burke B, Griffiths G, Reggio H, Louvard D, Warren G (1982) A monoclonal antibody against a $135-\mathrm{K}$ Golgi membrane protein. EMBO J 1:1621-1628

Chytilova E, Macas J, Sliwinska E, Rafelski SM, Lambert GM, Galbraith DW (2000) Nuclear dynamics in Arabidopsis thaliana. Mol Biol Cell 11:2733-2741

Cui P, Moreno Diaz De La Espina S (2003) Sm and U2B" proteins redistribute to different nuclear domains in dormant and proliferating onion cells. Planta 217:21-31

Darzacq X, Jady BE, Verheggen C, Kiss AM, Bertrand E, Kiss T (2002) Cajal body-specific small nuclear RNAs: a novel class of 2'-O-methylation and pseudouridylation guide RNAs. EMBO J 21:2746-2756

Deltour R (1985) Nuclear activation during early germination of the higher plant embryo. J Cell Sci 75:43-83

Deltour R, Mosen H, Bronchart R (1986) Three-dimensional electron microscopy of the internal nucleolus-associated chromatin and of the nucleolar vacuoles during early germination of Sinapis alba. J Cell Sci 82:53-71

Dundr M, Misteli T (2001) Functional architecture in the cell nucleus. Biochem J 356:297-310

Eils R, Gerlich D, Tvarusko W, Spector DL, Misteli T (2000) Quantitative imaging of pre-mRNA splicing factors in living cells. Mol Biol Cell 11:413-418

Gall JG (2000) Cajal bodies: the first 100 years. Annu Rev Cell Dev Biol 16:273-300

Gall JG (2001) A role for Cajal bodies in assembly of the nuclear transcription machinery. FEBS Lett 498:164-167

Graveley BR (2000) Sorting out the complexity of SR protein functions. RNA 6:1197-1211 
Hebert MD, Shpargel KB, Ospina JK, Tucker KE, Matera AG (2002) Coilin methylation regulates nuclear body formation. Dev Cell 3:329-337

Huang S, Deerinck T, Ellisman M, Spector D (1994) In vivo analysis of the stability and transport of nuclear poly(A)+ RNA. J Cell Biol 126:877-899

Jady BE, Darzacq X, Tucker KE, Matera AG, Bertrand E, Kiss T (2003) Modification of Sm small nuclear RNAs occurs in the nucleoplasmic Cajal body following import from the cytoplasm. EMBO J 22:1878-1888

Jennane A, Thiry M, Goessens G (1999) Identification of coiled body-like structures in meristematic cells of Pisum sativum cotyledonary buds. Chromosoma 108:132-142

Kevei E, Nagy F (2003) Phytochrome controlled signalling cascades in higher plants. Physiol Plant 117:305-313

Kircher S, Gil P, Kozma-Bognar L, Fejes E, Speth V, HusselsteinMuller T, Bauer D, Adam E, Schafer E, Nagy F (2002) Nucleocytoplasmic partitioning of the plant photoreceptors phytochrome A, B, C, D, and E is regulated differentially by light and exhibits a diurnal rhythm. Plant Cell 14:1541-1555

Kramer A (1996) The structure and function of proteins involved in mammalian pre-mRNA splicing. Annu Rev Biochem 65:367409

Lamond AI, Earnshaw WC (1998) Structure and function in the nucleus. Science 280:547-553

Lewis JD, Tollervey D (2000) Like attracts like: getting RNA processing together in the nucleus. Science 288:1385-1389

Lopato S, Waigmann E, Barta A (1996) Characterization of a novel arginine/serine-rich splicing factor in Arabidopsis. Plant Cell 8:2255-2264

Lopato S, Gattoni R, Fabini G, Stevenin J, Barta A (1999a) A novel family of plant splicing factors with a $\mathrm{Zn}$ knuckle motif: examination of RNA binding and splicing activities. Plant Mol Biol 39:761-773

Lopato S, Kalyna M, Dorner S, Kobayashi R, Krainer AR, Barta A (1999b) atSRp30, one of two SF2/ASF-like proteins from Arabidopsis thaliana, regulates splicing of specific plant genes. Genes Dev 13:987-1001
Lopato S, Forstner C, Kalyna M, Hilscher J, Langhammer U, Indrapichate K, Lorkovic ZJ, Barta A (2002) Network of interactions of a novel plant-specific Arg/Ser-rich protein, atRSZ33, with atSC35-like splicing factors. J Biol Chem 277:39989-39998

Lorkovic ZJ, Barta A (2002) Genome analysis: RNA recognition motif (RRM) and $\mathrm{K}$ homology $(\mathrm{KH})$ domain RNA-binding proteins from the flowering plant Arabidopsis thaliana. Nucleic Acids Res 30:623-635

Mas P, Devlin PF, Panda S, Kay SA (2000) Functional interaction of phytochrome B and cryptochrome 2. Nature 408:207-211

Matera AG (1999) Nuclear bodies: multifaceted subdomains of the interchromatin space. Trends Cell Biol 9:302-309

Misteli T (2000) Cell biology of transcription and pre-mRNA splicing: nuclear architecture meets nuclear function. J Cell Sci 113:1841-1849

Ogg SC, Lamond AI (2002) Cajal bodies and coilin - moving towards function. J Cell Biol 159:17-21

Platani M, Goldberg I, Lamond AI, Swedlow JR (2002) Cajal body dynamics and association with chromatin are ATP-dependent. Nat Cell Biol 4:502-508

Savaldi-Goldstein S, Aviv D, Davydov O, Fluhr R (2003) Alternative splicing modulation by a Lammer kinase impinges on developmental and transcriptome expression. Plant Cell 15:926-938

Shaw PJ (1996) Nuclear organization in plants. Essays Biochem $31: 77-89$

Sleeman JE, Ajuh P, Lamond AI (2001) snRNP protein expression enhances the formation of Cajal bodies containing p80-coilin and SMN. J Cell Sci 114:4407-4419

Straatman KR, Schel JH (2001) Distribution of splicing proteins and putative coiled bodies during pollen development and androgenesis in Brassica napus L. Protoplasma 216:191-200

Testillano PS, Sanchez-Pina MA, Olmedilla A, Fuchs JP, Risueno MC (1993) Characterization of the interchromatin region as the nuclear domain containing snRNPs in plant cells. A cytochemical and immunoelectron microscopy study. Eur J Cell Biol 61:349-361 